\title{
Dual Mode Scramjet Design to Achieve Improved Operational Stability
}

\author{
Sean M. Torrez, Derek J. Dalle, James F. Driscoll ${ }^{\dagger}$ \\ University of Michigan, Ann Arbor, MI 48109
}

\begin{abstract}
Control evaluation and multidisciplinary optimization are two types of computation that require extremely fast computation of vehicle or component performance. These computations require relatively accurate prediction of performance and performance trends, but they do not need to retain full-fidelity information about every part of the vehicle. This paper presents some results of a computer code that predicts the performance of scramjet and ramjet powered vehicles. The code (including some rudimentary design capability) runs in less than 2 seconds. Run times for batch analysis can be faster because redesign is not required at each iteration. Thermodynamic performance traces are presented along the flow path length. Combustor performance and design are analyzed with respect to performance and stability over a range of operating conditions. Sensitivity plots of thrust with respect to Mach number and altitude are shown. Operating maps are presented for comparison between different proposed designs.
\end{abstract}

\section{Nomenclature}
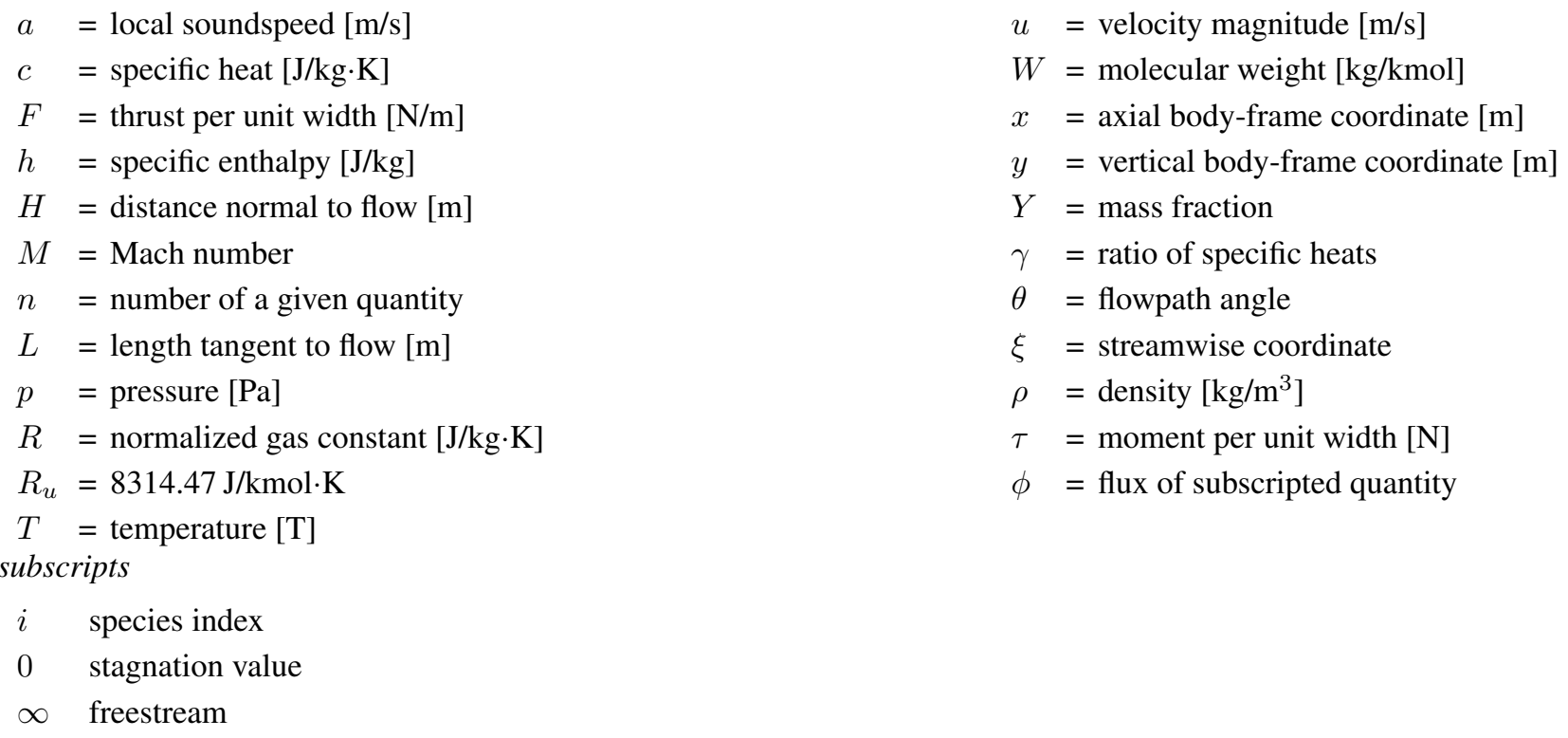

\section{Introduction}

Scramjet and ramjet combustors differ from conventional gas turbine combustors because their geometric compression components can have fundamentally different performance depending on the thermodynamic and chemical characteristics of the incoming flow. ${ }^{1}$ This means that the central question is not just how much of the fuel burns, but where and how the fuel burns. ${ }^{2}$ The MASIV code presented here predicts the performance of an entire vehicle flow

\footnotetext{
* Graduate Research Assistant, Department of Aerospace Engineering, AIAA Student Member
}

${ }^{\dagger}$ Professor, Department of Aerospace Engineering, AIAA Fellow 
path, including inlet, isolator, combustor, and nozzle. Some basic design methodologies are applied to each component so that for a given choice of design point a full vehicle flowpath geometry is drawn.

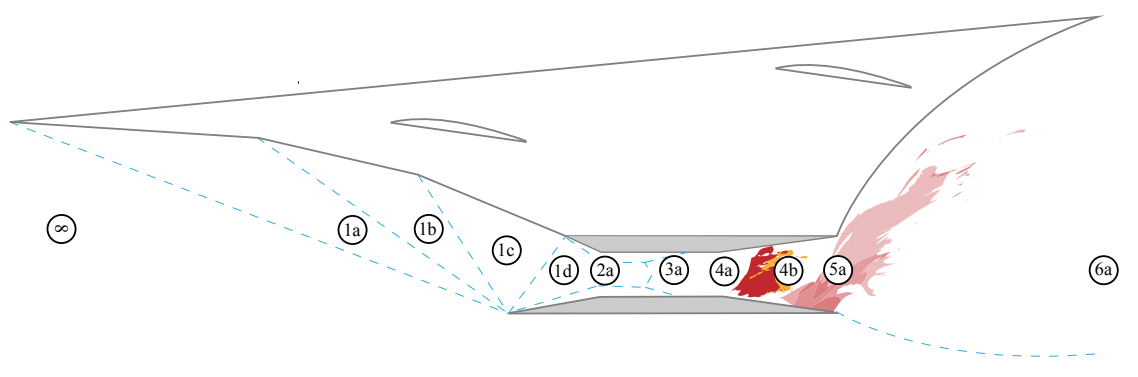

Figure 1. An example vehicle geometry with extensible station numbering.

The goal in these simulations is not to replace high-fidelity and high-spatial-resolution CFD solutions or realworld experiments. Instead, we seek to match CFD and experimental results to an adequate degree of accuracy while minimizing the computation time for the full flow path to keep it on the order of $1 \mathrm{~s}$. Here, an adequate degree of accuracy means that for a user-specified total error (usually in terms of thrust $F$, lift $L$, or moment $M$ ), the acceptable error for each component must fall below a certain threshold. The results of a reduced-order model (ROM) such as MASIV are useful for multidisciplinary optimization (MDO) ${ }^{3}$ and control design and evaluation ${ }^{4}$ because they accurately reproduce the performance trends and values expected from a given vehicle design, but they do not spend computation time on those aspects of the solution that are not directly involved in the computation of $F, L$ or $M$.

In developing a ROM to satisfy these goals, we have followed an approach that restricts the size of the physical problem at hand, ${ }^{5,6}$ rather than simply computing a large table of vehicle performance over a range of operating conditions. We limit the dimension of the physical problem as much as possible and then solve the problem directly for each flow path evaluation. This means that upon use for MDO or control design and evaluation the code is not limited by pre-specified bounds, as a performance table would be. We can compute performance at a desired operating condition directly.

The development of the individual sub-models that comprise the MASIV code has been sufficiently discussed elsewhere, ${ }^{5,7}$ so only a brief discussion is warranted here. The reader may consult these past references for the details. The inlet and nozzle solutions are both computed by a 2-D wave method which solves directly for the flow conditions in each region by approximating the positions of shocks and expansions and treating expansions as a number of discrete waves. Interactions between waves are solved as two-dimensional Riemann problems. This is similar to a method of characteristics.

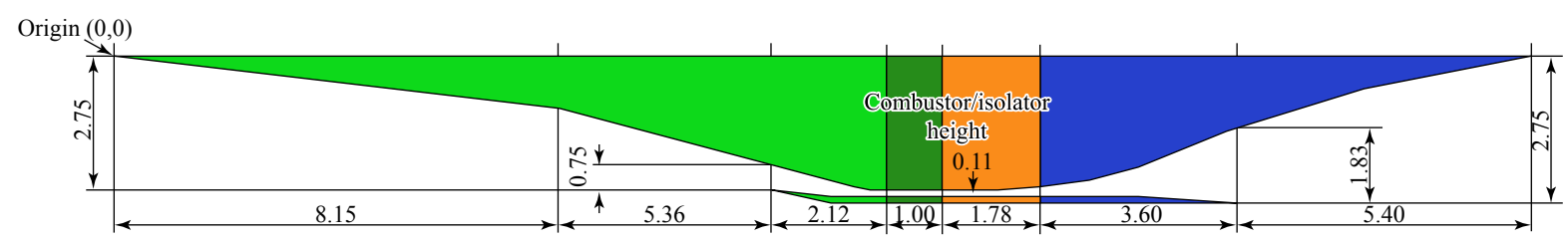

Figure 2. The baseline design vehicle considered in this study. All dimensions are in meters.

The combustor flow-field is solved by combining a three-dimensional jet mixing model with a flamelet chemistry solver. These models provide a one-dimensional reaction rate for each species involved, which is then integrated along with a set of ODEs that governs the evolution of the thermodynamic state variables in the duct. This is similar to CFD methods in which reaction rates are determined by a subgrid-scale modeling approach. Solving ODEs rather than PDEs for the flow field is appropriate for ram- and scramjet conditions because the flow path is mostly supersonic and molecular diffusion in the direction of flow is relatively unimportant.

Our initial studies ${ }^{8}$ of hypersonic vehicle design have shown that one major concern is that the operating point provide adequate vehicle stability and that the operating point curves be sufficiently smooth. Vehicle stability is related to control stability in the sense that adequate performance should be maintained even when the operating point is displaced from the design point. A "smooth" design point means that for small perturbations from the design point performance perturbations will also be small. Clearly, these two considerations are related but are not the same. A good design ought to satisfy both of them. Usually, there is some tradeoff between design point performance and the 
overall operating range, meaning that peak performance will be reduced in order to guarantee stability and smoothness over a range of conditions.

The station numbering we have chosen for hypersonic vehicles is shown in Fig. 1. An example of the baseline flowpath geometry designed using our algorithm is shown in Fig. 2. This geometry is designed to operate over a range of conditions rather than a single operating point, as will be discussed. Note that this is only an engine flowpath geometry and that the full-vehicle geometry is not treated in this study.

\section{Design Methodology}

Our principal goal for the design of the hypersonic vehicle flow path was to ensure good performance over the expected range of operating conditions, which we define as a certain range around the nominal design point. We select a vehicle with a nominal operating point of $M=8.0$, altitude of $26000 \mathrm{~m}$ and we design an inlet to have acceptable performance over the Mach number range from 7.0 to 9.0 , meaning that there are no serious undesirable wave interactions causing poor thermodynamic performance. If this is the case, the flow delivered to the isolator will also fall within a reasonable range and it will be possible to design the combustor for a known range of conditions.

For this study, we consider only scram combustors, so we require that the flow remains supersonic through all parts of the flow path. The combustor is the component with the greatest chance of violating this condition because heat addition drives the Mach number toward unity. The basic requirement, then, for a successful scram combustor is that the flow does not choke. A competing requirement for good performance is that the pressure rise in the combustor is maximized, since this will give greater thrust. We establish some safety factor for this low- $M$, high- $p$ requirement by specifying a minimum design Mach number in the combustor of 1.5.

Table 1 shows some of the parameters of the flow path that cannot be seen directly from the geometric profile.

Table 1. Flow Path Design Specifications

\begin{tabular}{r|c|c}
\hline \hline Design Variable & Value & Units \\
\hline Length & 1.78 & $\mathrm{~m}$ \\
Number of Injectors & 12 & $\frac{1}{\mathrm{~m}}$ \\
Injector Diameter & 3.73 & $\mathrm{~cm}$ \\
Internal Contraction Ratio & 4.28 & - \\
Compression Ratio $\left(p_{2} / p_{\infty}\right)$ & 50.3 & - \\
\hline
\end{tabular}

\section{A. Inlet}

A reduced order model of the 2-D supersonic has been discussed previously. ${ }^{?}$ The references provide more information.

\section{B. Isolator}

The main concern in the isolator is that the shock pattern that occurs during subsonic combustion operation does not reach the inlet since this condition can cause the engine to unstart. ${ }^{9}$ For high supersonic flight Mach numbers (and correspondingly high combustor Mach numbers), the boundary layers in the isolator will be very thin and no waves of any importance will form in the isolator. In this study we consider only the scram conditions around a Mach 8 baseline case so we do not consider the isolator in detail. Instead, we "design" the isolator by using the rule of thumb that its length must be 6 times as great as its height. ${ }^{10}$

\section{Combustor}

To reiterate, combustors (as well as inlets) must be designed for a range of input conditions if they are to function properly. This means we must select the expected heat release profile such that it will not choke in the scram mode and will only choke at the desired point in ram mode. It seems that this may be accomplished almost entirely through geometric (passive) means, although in some cases active fuel scheduling may be required.

Here, we consider a typical cavity-stabilized combustor since most proposed designs are of this type. It is not necessary to consider the cavity in predicting thrust at this level of fidelity because the flow in the cavity is reversed, 
meaning that the main flow cannot penetrate very far at all into the cavity. This in turn means that most combustors that operate in a diffusion flame mode consist of a line of injectors some distance away from a diverging section. The placement of the injectors with reference to the beginning of the divergence determines whether the flow will choke and where it will choke as the fuel addition rate is increased. The angle of divergence of the combustor can also influence the onset of choking because it can keep the flow supersonic in regions of heat addition by allowing the flow to expand.

\section{Nozzle}

Qualitatively, nozzles typically operate successfully over a range of conditions without any specific design optimization, since they self-adjust to some extent. ${ }^{11,12}$ It is unlikely that a nozzle that is well-designed at one condition would have poor performance at any reasonable off-design condition. Any waves that the nozzle generates will not interact with the vehicle itself, and will interact with each other far behind the vehicle, which will not affect the thrust generated. This results in a nozzle that is already smooth for most reasonable ranges of operating conditions.

\section{Performance Calculation}

The details of the flow path performance calculations are given in papers by Torrez et al. ${ }^{6,7}$ and Dalle et al. ${ }^{5}$ Here we discuss some speed improvements for calculation of propulsion performance.

MASIV computes the evolution of thermodynamic variables and mass fractions of flow species throughout the duct. Hence, the state variable is $\phi=[\rho, p, T, u, \theta, Y]$, where $\theta$ is the flow angle with respect to the vehicle centerline and $Y$ is the vector of species mass fractions. Each component uses either 3-D or 2-D techniques in order to generate 1-D profiles of the state, which then gives a 1-D profile for the entire engine flow path.

The 3-D and 2-D information that is generated must be integrated across planes throughout the flow path in order to generate the 1-D profile. This is accomplished in the combustor by applying an interpolation scheme to the pretabulated reaction rate information and then integrating using a conventional trapezoidal method. The resulting 1-D rate profile is then integrated as part of a set of ODEs. This requires first that we interpolate between the computed reaction rate information, which is computed along lines perpendicular to the flame center line. First, we take a weighted average on each line for each of the interpolation points (see Fig. 3). We assume that $(x, y)$ is the position of the interpolation point, while $(x, y)_{\text {left }}$ and $(x, y)_{\text {right }}$ are the coordinates of the nearest computed reaction points to the left and right, respectively, along the perpendicular lines

$$
\begin{aligned}
d_{\text {left } / \text { right }} & =\sqrt{\left(x-x_{\text {left } / \text { right }}\right)^{2}+\left(y-y_{\text {left } / \text { right }}\right)^{2}} \\
d_{\text {total }} & =\sqrt{\left(x_{\text {right }}-x_{\text {left }}\right)^{2}+\left(y_{\text {right }}-y_{\text {left }}\right)^{2}} \\
c_{\text {left } / \text { right }} & =\frac{d_{\text {left } / \text { right }}}{d_{\text {total }}} \\
f & =\sum_{\text {left } / \text { right }} c_{\text {left } / \text { right }} f_{\text {left } / \text { right }}
\end{aligned}
$$

where $f$ is the value of the reaction rate function at the interpolation point, and $f_{\text {left/right }}$ are the reaction rates at the points to the left and right of the desired one. Once the interpolation has been completed, the points can be integrated in the $y$-direction using any desired scheme.

In the inlet and the nozzle, there are definite 2-D regions, rather than lines, which define the flow properties. The 1-D value at a given point can be found by performing a mass-weighted average of all the regions through which a vertical line corresponding to a given $x$-point passes. The length of the line through each region is found by the vertical distance between intersections with region boundaries. Then, the weights are determined by dividing each height by the total height and multiplying by the density in that region

$$
\begin{array}{r}
w_{i}=H_{i} \rho_{i} \\
\phi=\sum w_{i} \phi_{i}
\end{array}
$$

where $i$ denotes each region that overlaps the chosen $x$-point, $H_{i}$ is the height of each region, and $\phi$ is the state vector.

The inlet and nozzle codes use method-of-characteristics type solutions, ${ }^{5}$ so the values given by this interpolation are the final values needed to compute the force and moment for each component. In the combustor, this 1-D reaction 


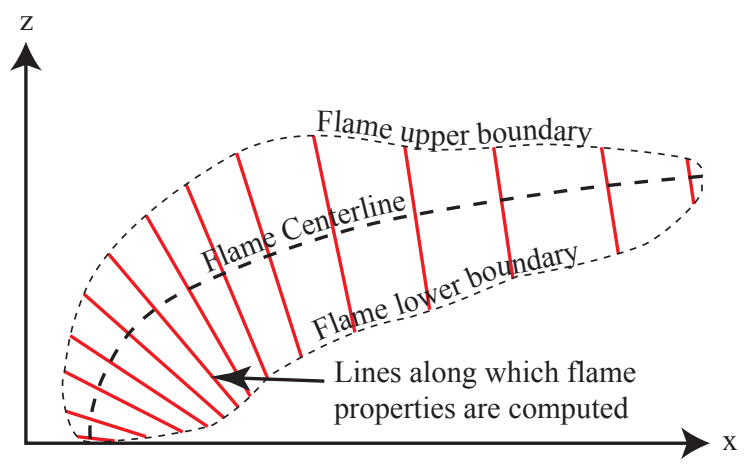

a) Computed flame reaction rate lines

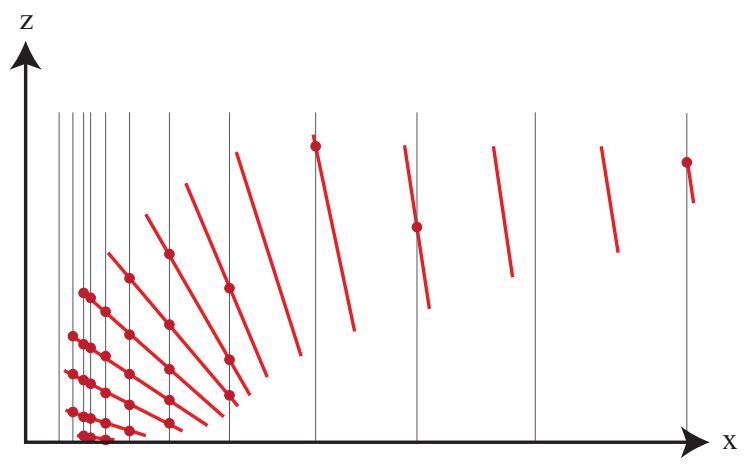

b) Points interpolated onto chosen $x$-points

Figure 3. Interpolation scheme for the combustor. Note that the non-uniform spacing automatically places more points near the injector, where most of the heat release occurs.

rate information is integrated as part of a set of ODEs, ${ }^{7}$ which gives 1-D thermodynamic information. Forces and moments per unit width are calculated by using the stream-tube reaction thrust equation. For thrust, the equation is:

$$
\begin{aligned}
F & =\dot{m}_{3} u_{3}-\dot{m}_{5} u_{5}+p_{3} H_{3}-p_{5} H_{5} \\
& =\rho_{3} u_{3}^{2} H_{3}-\rho_{5} u_{5}^{2} H_{5}+p_{3} H_{3}-p_{5} H_{5}
\end{aligned}
$$

The states 3 and 5 represent the conditions before and after the combustor, respectively. The moment equation can also be calculated algebraically, since we assume that conditions are uniform across the height of the flow path. This gives the moment equation

$$
\tau=\frac{1}{2} H_{3}^{2}\left(p_{3}+\rho_{3} u_{3}^{2}\right)-\frac{1}{2} H_{5}^{2}\left(p_{5}+\rho_{5} u_{5}^{2}\right)-H_{e} H_{5}\left(p_{5}+\rho_{5} u_{5}^{2}\right)
$$

where $\tau$ is the moment per unit width generated by each component (in this case the combustor) about its own leading top edge and $H_{e}$ is the height of the combustor exit area that falls above the leading top edge. Therefore, the first term represents the moment (positive counter-clockwise) generated by the momentum flux at the front of the combustor, the second term represents the force generated by the momentum flux at the rear and the third term represents the added moment created by the force at the rear being applied at a point not in line with the leading top edge of the combustor.

\section{Performance Results}

There are two important considerations as far as vehicle performance is concerned. The flow path should provide near-optimum performance at design conditions, but nowhere in the design range should the performance be severely degraded. We desire that changes in vehicle performance (thrust) should be smooth for a change in altitude or Mach number.

Design point performance characteristics are shown in Table 2. Note that the flow path is designed for an equivalence ratio of $\phi=0.5$. The operating point properties are listed in Table 3. Note that the performance plots shown in Fig. 4 through Fig. 9 are for equivalence ratio of $\phi=0.3$. This is consistent the requirement that the vehicle be able to accelerate from the operating point.

\section{A. Nominal Performance}

Since the figure of merit for our scramjet flowpath design is smoothness and stability over the design range, our assessment of the engine design includes evaluating its sensitivity to variations in the operating point. For example, Fig. 5 shows the sensitivity of thrust with respect to $M_{\infty}$ and altitude as the design and the equivalence ratio are kept constant. This is a way of considering rapid variations in $M$ or altitude that an airplane might see while in flight due to gusts. A gust could be considered a type of disturbance in this situation. 


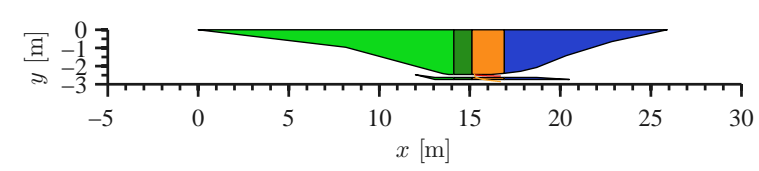

a) Vehicle geometry - for comparison

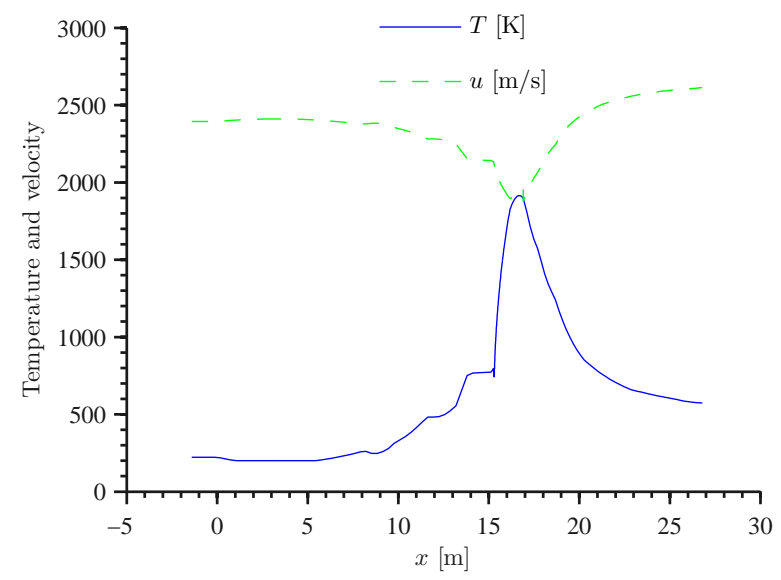

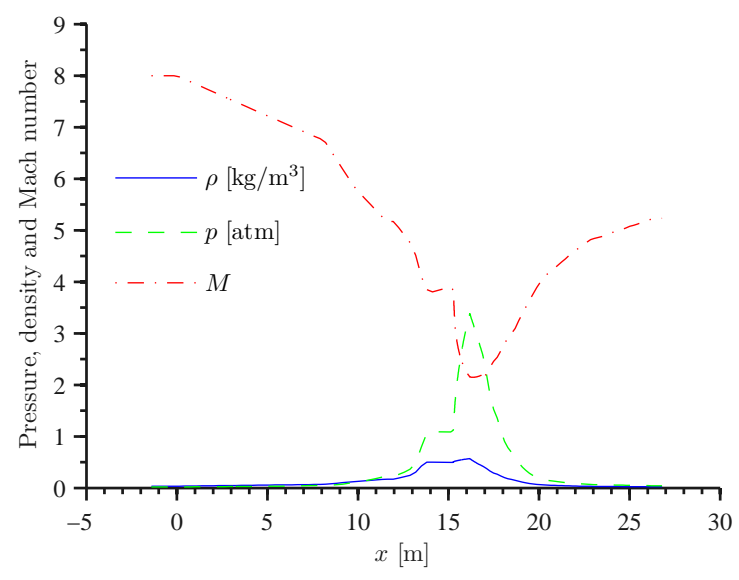

b) Pressure, density and Mach number throughout the flow path for the design point $(M=8, \alpha=0$, altitude $=26000 \mathrm{~m}, \phi=0.5)$

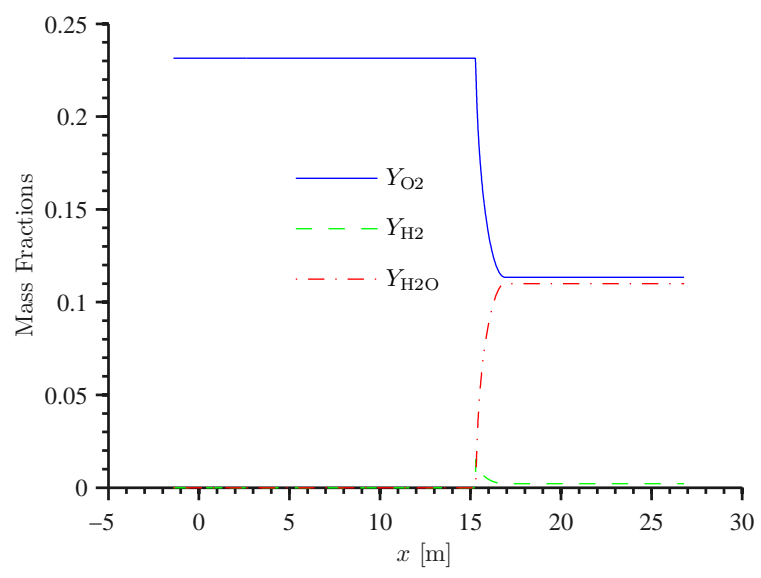

c) Temperature and velocity throughout the flow path for the design d) Mass fractions throughout the flow path for the design condition. condition.

Figure 4. Full flowpath evolutions of state quantities at design.

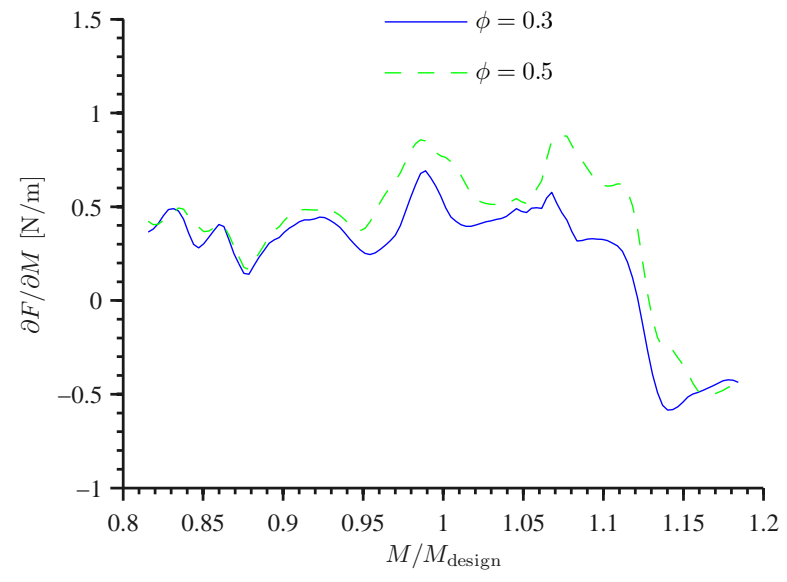

a) Design point sensitivity to Mach number variation

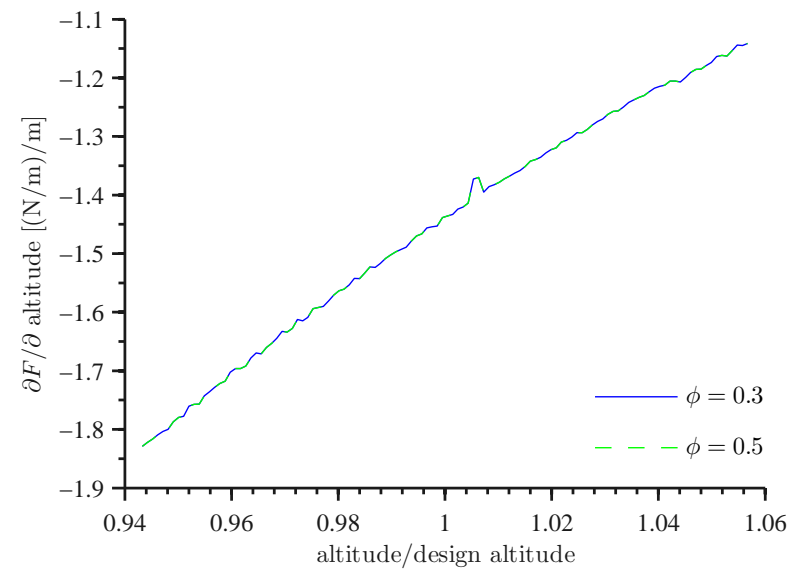

b) Design point sensitivity to altitude variation

Figure 5. Derivatives of thrust per unit width with respect to $M$ and altitude, at full design throttle $(\phi=0.5)$ and $60 \%$ design throttle $(\phi=0.3)$. 
Table 2. Engine Design Point Performance Characteristics

\begin{tabular}{r|c|c}
\hline \hline Design Variable & Value & Units \\
\hline Design Equivalence Ratio & 0.5 & - \\
Design Fuel Mass Flow Rate & 2.84 & $\frac{\mathrm{kg}}{\mathrm{s}}$ \\
Design Air Mass Flow Rate & 177 & $\frac{\mathrm{kg}}{\mathrm{s}}$ \\
Compression Ratio $\left(p_{2} / p_{\infty}\right)$ & 50.3 & - \\
Pressure Recovery Factor & 63.0 & - \\
Thrust & 52.0 & $\frac{\mathrm{kN}}{\mathrm{m}}$ \\
Specific Impulse & 1870 & $\mathrm{~s}$ \\
Specific Thrust & 0.294 & $\frac{\mathrm{kN}}{\mathrm{kg} / \mathrm{s}}$ \\
\hline \hline
\end{tabular}

Note that around the design point and throughout the design range there are no points for which the thrust changes rapidly (Figure 6). The sensitivity is low in general, but the sensitivity does not change rapidly either, meaning that this flow path can sustain changes in its operating point without giving a disproportionately large change in thrust. The operating point characteristics are shown in Table 3. Of course, the thrust produced at $60 \%$ throttle is much lower than at $100 \%$, so we are making the assumption that the vehicle only requires $36400 \mathrm{kN} / \mathrm{m}$ of thrust to balance drag.

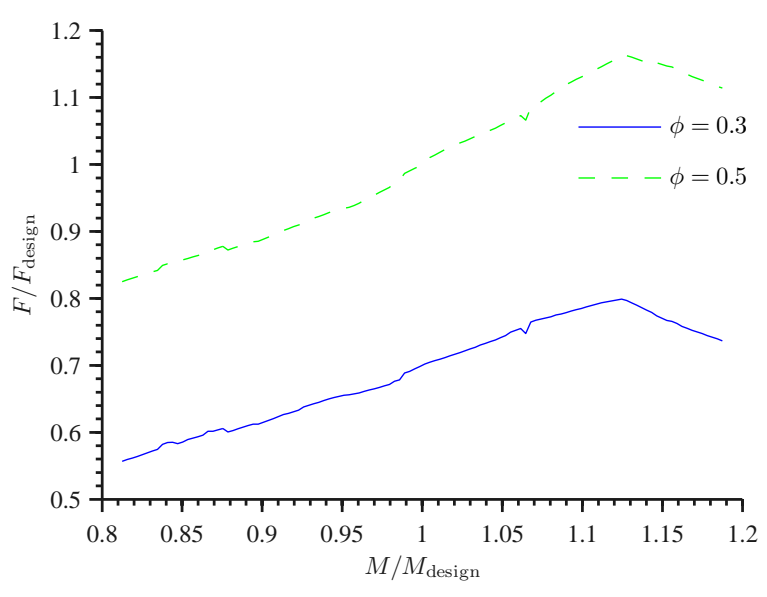

a) Thrust as a function of Mach number

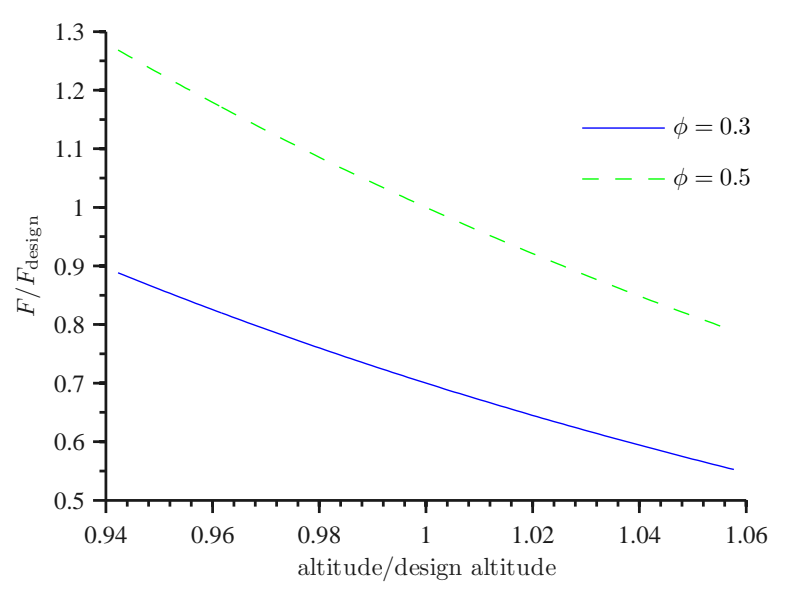

b) Thrust as a function of altitude

Figure 6. Thrust curves with respect to altitude and Mach number.

Table 3. Engine Operating Point Performance Characteristics

\begin{tabular}{r|c|c}
\hline \hline Design Variable & Value & Units \\
\hline Operating Equivalence Ratio & 0.3 & - \\
Operating Fuel Mass Flow Rate & 1.70 & $\frac{\mathrm{kg}}{\mathrm{s}}$ \\
Operating Air Mass Flow Rate & 177 & $\frac{\mathrm{kg}}{\mathrm{s}}$ \\
Compression Ratio & 50.3 & - \\
Pressure Recovery Factor & 63.0 & - \\
Thrust & 36.4 & $\frac{\mathrm{kN}}{\mathrm{m}}$ \\
Specific Impulse & 2180 & $\mathrm{~s}$ \\
Specific Thrust & 0.206 & $\frac{\mathrm{kN}}{\mathrm{kg} / \mathrm{s}}$ \\
\hline \hline
\end{tabular}

The sensitivity of thrust with respect to Mach number goes down as equivalence ration $\phi$ is reduced because the engine generates less thrust under the lower throttle setting. Figure 5 also indicates that at a partial throttle setting, the 
peaks and valleys in the sensitivity are reduced compared to the mean. This would indicated some benefit for control purposes because the airplane is expected to operate under partial throttle for cruise. Figure 7 shows the full flowpath evolution of state quantities for the $\phi=0.3$ case. Comparing this to the $\phi=0.5$ case, a possible explanation of this reduced sensitivity is that the minimum Mach number seen in the combustor is lower at partial throttle than at full throttle. This in turn means that the maximum static pressure is reduced, reducing the pressure ratio due to reaction, reducing the thrust generated due to Mach number variations. So, if we design to place the minimum Mach number at 1.5 at the edge of the design range, all other operating points within that range should have higher minimum Mach number and reduced sensitivity to flight Mach number variations.

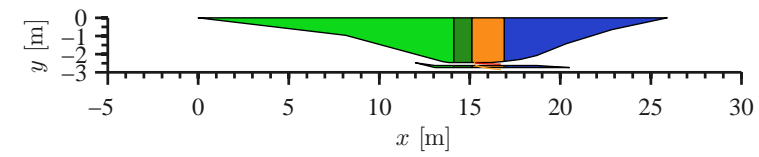

a) Vehicle geometry - for comparison

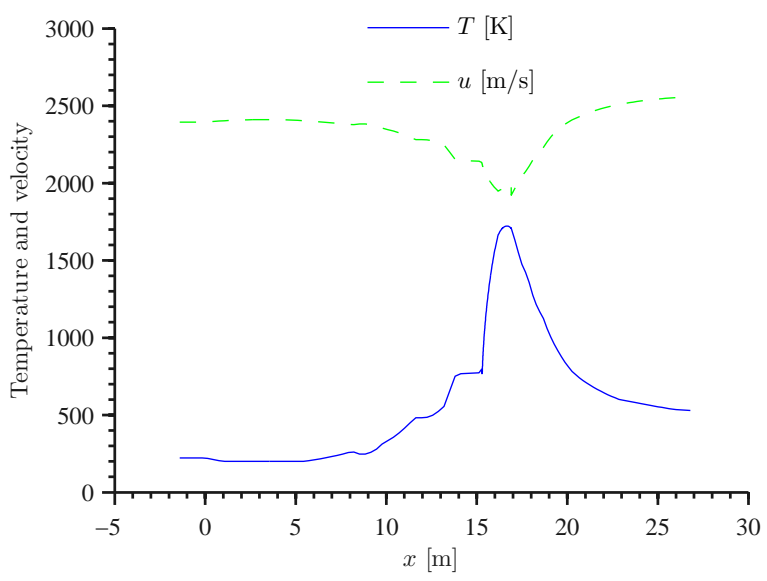

c) Temperature and velocity throughout the flow path for the nominal operating condition.

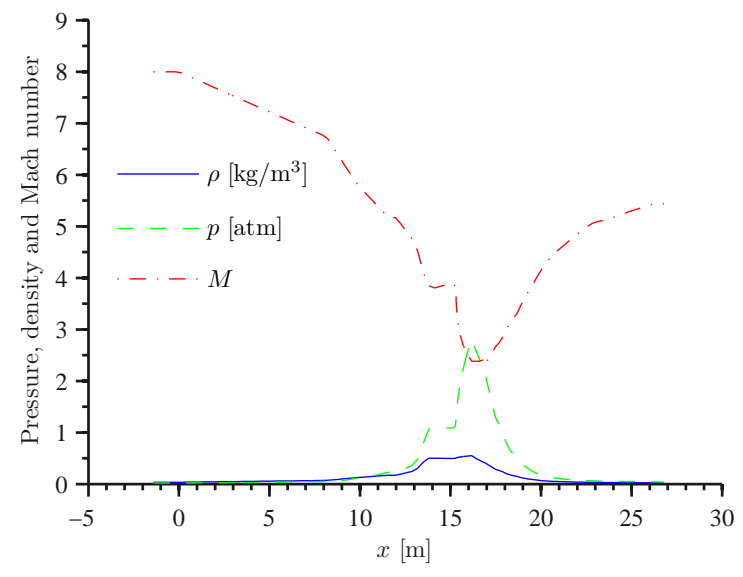

b) Pressure, density and Mach number throughout the flow path for the nominal operating condition $(M=8, \alpha=0$, altitude $=26000$, $\phi=0.3)$

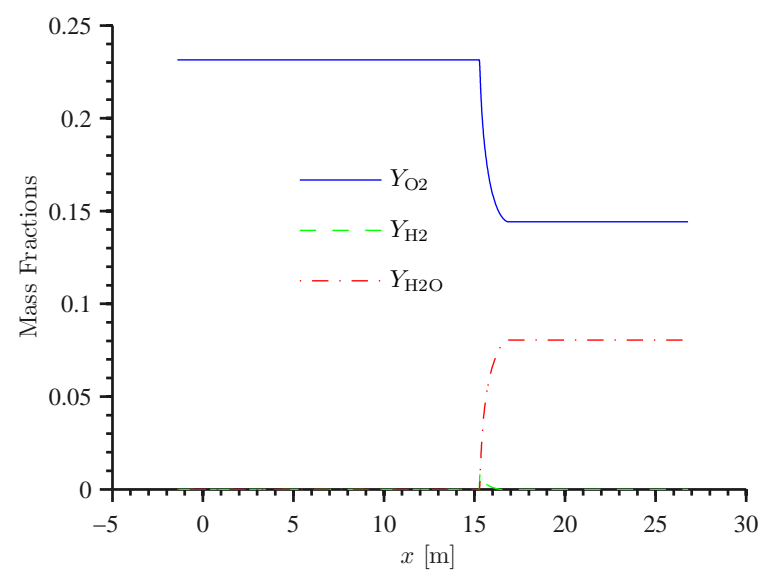

i- d) Mass fractions throughout the flow path for the nominal operating condition.

Figure 7. Full flowpath evolutions of state quantities, $60 \%$ throttle.

\section{B. Off-Design Performance}

We consider two off-design conditions. These correspond to the lower-left corner of the design range $(M=7$, altitude $=25000 \mathrm{~m})$ and the upper-right corner of the design range $(M=9$, altitude $=27000 \mathrm{~m})$. We require that the engine operates and generates thrust at full throttle at each of these conditions, subject to the previously mentioned Mach number safety factor. Figure 8 and Fig. 9 show the full-flowpath evolutions of the state variables at these two conditions.

The upper-right corner condition does not encounter serious problems, because the flight Mach number is high enough to prevent the Mach number in the combustor from getting dangerously low. This assumes that the fuel 
injection scheme is rapidly able to respond to changes in air mass flow rate in order to keep the equivalence ratio constant.

The lower-left corner condition is really the one that sets the most major design constraint, since it appears to be here that the combustor will have the highest likelihood of choking. The combustor is designed to have $M=1.5$ at $100 \%$ throttle at this condition. This means that at $60 \%$ throttle, the Mach number only drops to about 2 , a fairly safe number.

For off design conditions, then, we identify the most important consideration for scram to be that of keeping the engine operating in the scram range. In this way, we can prescribe a range for which the engine must always operate in scram and transition to ram only when appropriate.

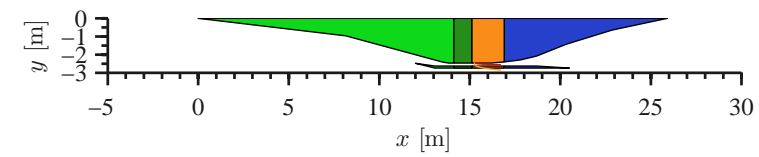

a) Vehicle geometry - for comparison

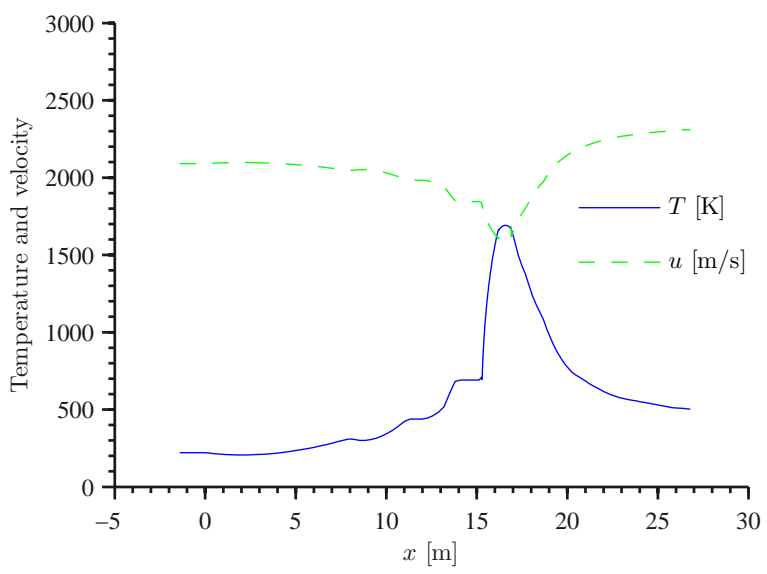

c) Temperature and velocity throughout the flow path for the low speed, low altitude limit.

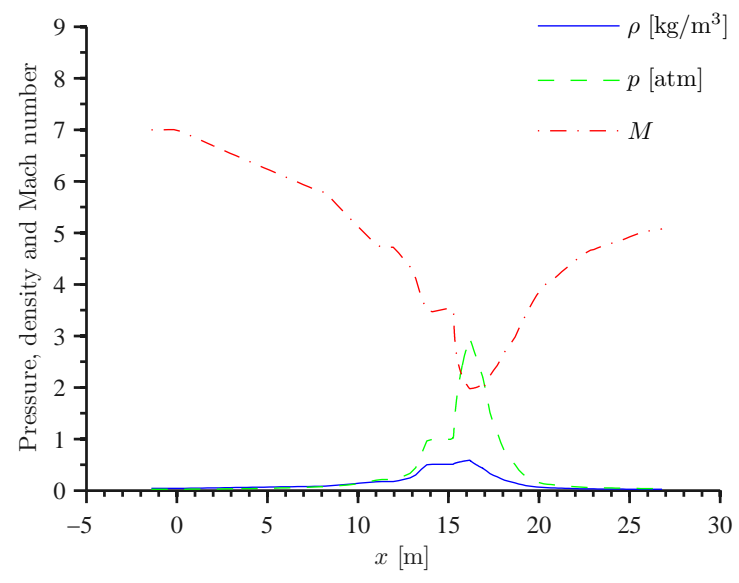

b) Pressure, density and Mach number throughout the flow path for the low speed, low altitude limit $(M=7, \alpha=0$, altitude $=25000$, $\phi=0.3)$

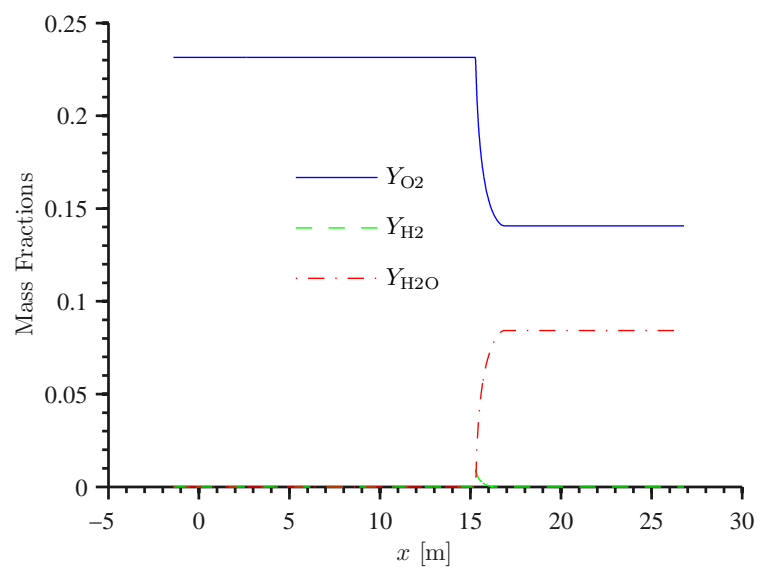

d) Mass fractions throughout the flow path for the low speed, low altitude limit.

Figure 8. Full flowpath evolutions of state quantities at lowest operating point.

\section{Operation Maps}

Operation maps for $0.1<\phi 0.6$ are shown in Fig. 10. We observe the expected trends of thrust increasing as mass flow rate increases, both as density increases with altitude and as velocity increases with Mach number. The wavy nature of the curves arises because the precise amount of mass flow through the engine changes slightly as the shock pattern in the inlet changes with flight Mach number. Of course, the thrust numbers on each plot increase as equivalence ratio increases. Note that in all of the plots the thrust curves trend to horizontal at the lower Mach number limit.

These plots represent thrust generated at a constant throttle setting, because we only consider rapid deviations from 

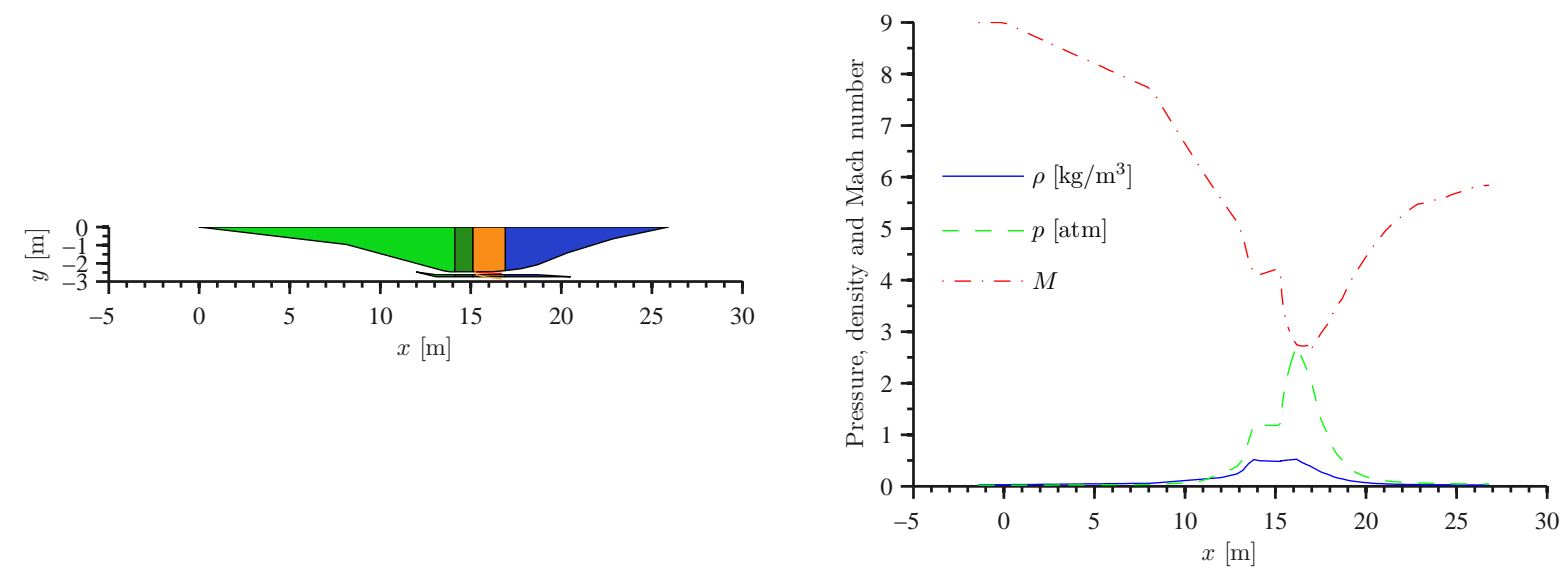

a) Vehicle geometry - for comparison

b) Pressure, density and Mach number throughout the flow path for the high speed, high altitude limit $(M=9, \alpha=0$, altitude $=27000$, $\phi=0.3)$
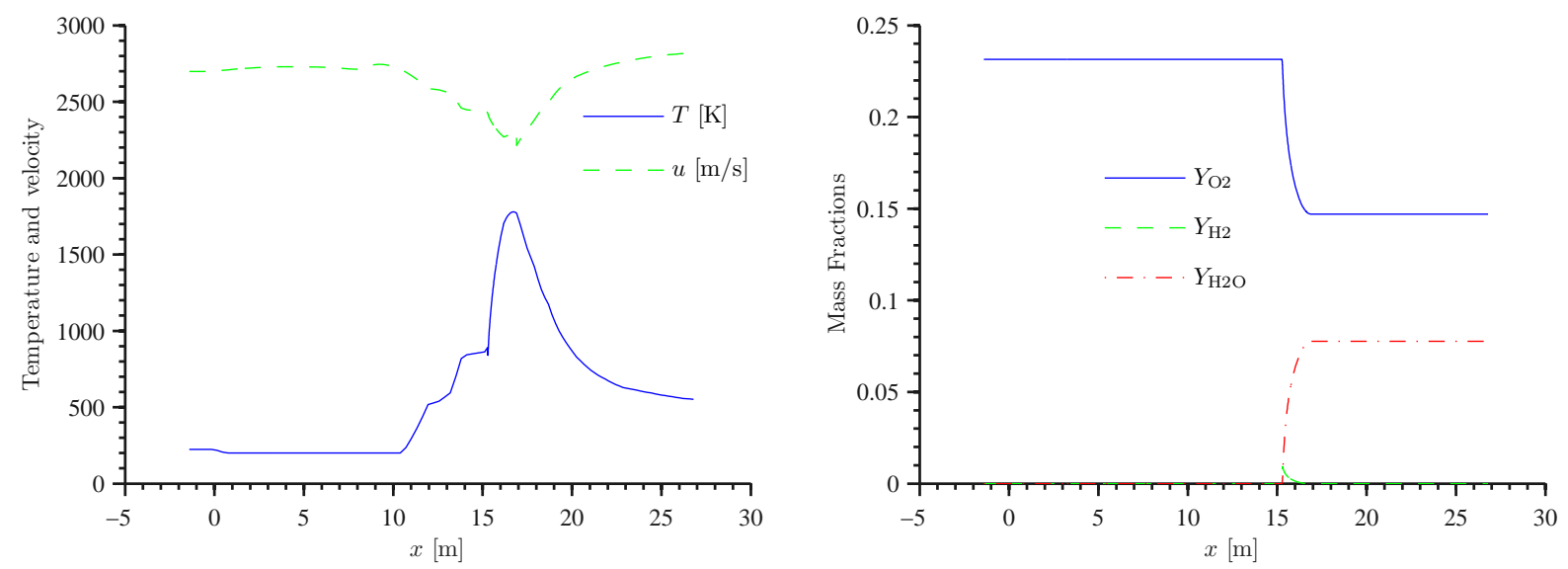

c) Temperature and velocity throughout the flow path for the high d) Mass fractions throughout the flow path for the high speed, high speed, high altitude limit. altitude.

Figure 9. Full flowpath evolutions of state quantities at highest operating point. 
the nominal operating point. The flow path would of course require a higher equivalence ratio to trim the vehicle for the lower Mach number and altitude conditions due to lower mass flow rate through the engine.

\section{Conclusions}

A basic design methodology for hypersonic engine flowpaths has been presented which focuses on avoiding choking conditions to maintain thrust throughout a design space. Secondary, but still important to the design, is the generation of maximum pressure rise in the combustor because this leads to maximum thrust generation. The design methodology has been used to create a 2-D vehicle flow path design, which was then analyzed using the MASIV code to show performance characteristics over the design range. This analysis shows that smooth variation of thrust with respect to Mach number and altitude can be achieved, mostly through careful inlet design.

Combustor design also influences the thrust a great deal, because the combustor fuel injection location and geometric expansion profile govern the choking limit and the amount of pressure rise generated by combustion. This paper presents some initial guidelines and concerns for combustor design and a framework within which designs can be compared. More work is needed to determine some more robust guidelines for combustor expansion profiles and injection location and angle, as well as possibilities for anchoring the choking location when transition between scramand ram-mode is considered.

The configuration shown is predicted to produce thrust over the design range and to have performance without serious deficiencies or rapid changes in thrust throughout the range. However, the profile of $\partial F / \partial M$ is of possible concern because it would be difficult to build a controller to handle such a poorly-behaved function. There are also some identified points in several of the operating maps for which the model predicts degraded performance. More work is needed to determine why the given combustor design chokes and how to ensure that choking only occurs at prescribed locations and under prescribed conditions.

\section{Acknowledgements}

This research was supported by AFRL grant FA 8650-07-2-3744 for the MACCCS (Michigan AFRL Collaborative Center for Control Science) and by NASA grant NNX08AB32A, administered by Donald Soloway, technical monitor. Portions of the work were done in collaboration with M. Bolender and D. Doman of the Air Force Research Lab.

\section{References}

\footnotetext{
${ }^{1}$ Mathur, T., Streby, G., Gruber, M., Jackson, K., Donbar, J., Donaldson, W., and Jackson, T., "Supersonic Combustion Experiments with a Cavity Based Fuel Injector," 1999, AIAA 99-2102.

${ }^{2}$ Micka, D. J., Torrez, S. M., and Driscoll, J. F., "Measurements and Modeling of the Heat Release Distribution in a Dual-mode Scramjet Combustor with Wall Fuel Injection," Proceedings of the 6th U.S. National Combustion Meeting, 2009.

${ }^{3}$ Bowcutt, K. G., "Multidisciplinary Optimization of Airbreathing Hypersonic Vehicles," Journal of Propulsion and Power, Vol. 17, 2001, pp. 1184-1190.

${ }^{4}$ Bolender, M. A. and Doman, D. B., "Nonlinear Longitudinal Dynamical Model of an Air-Breathing Hypersonic Vehicle," Journal of Spacecraft and Rockets, Vol. 44, No. 2, 2007, pp. 374-387.

${ }^{5}$ Dalle, D. J., Fotia, M. L., and Driscoll, J. F., "Reduced-Order Modeling of Two-Dimensional Supersonic Flows with Applications to Scramjet Inlets," Journal of Propulsion and Power, Vol. 26, No. 3, 2010, pp. 545-555.

${ }^{6}$ Torrez, S. M., Driscoll, J. F., Ihme, M., and Fotia, M. L., "Reduced Order Modeling of Turbulent Reacting Flows With Application to Scramjets," Journal of Propulsion and Power, 2010, To Appear.

${ }^{7}$ Torrez, S. M., Driscoll, J. F., Dalle, D. J., and Micka, D. J., "Scramjet Engine Model MASIV: Role of Mixing, Chemistry and Wave Interaction," 45th AIAA/ASME/SAE/ASEE Joint Propulsion Conference and Exhibit, 2009, AIAA Paper No. 2009-4939.

${ }^{8}$ Torrez, S. M., Driscoll, J. F., Dalle, D. J., and Fotia, M. L., "Preliminary Design Methodology for Hypersonic Engine Flowpaths," 16th AIAA/DLR/DGLR International Space Planes and Hypersonic Systems and Technologies Conference, 2009.

${ }^{9}$ Heiser, W. H. and Pratt, D. T., Hypersonic Airbreathing Propulsion, AIAA Pub., Washington, DC, 1994.

${ }^{10}$ Le, D. B., Goyne, C. P., Krauss, R. H., and McDaniel, J. C., "Experimental Study of a Dual-Mode Scramjet Isolator," 2005, AIAA $2005-23$.

${ }^{11}$ Rao, G. V. R., "Recent Developments in Rocket Nozzle Configurations," ARS Journal, 1961, pp. 1488-1494.

${ }^{12}$ Korte, J. J., Salas, A. O., Dunn, H. J., Alexandrov, N. M., Follett, W. W., Orient, G. E., and Hadid, A. H., "Multidisciplinary Approach to Linear Aerospike Nozzle Design," Journal of Propulsion and Power, Vol. 17, No. 1, 2001, pp. 93-98.
} 


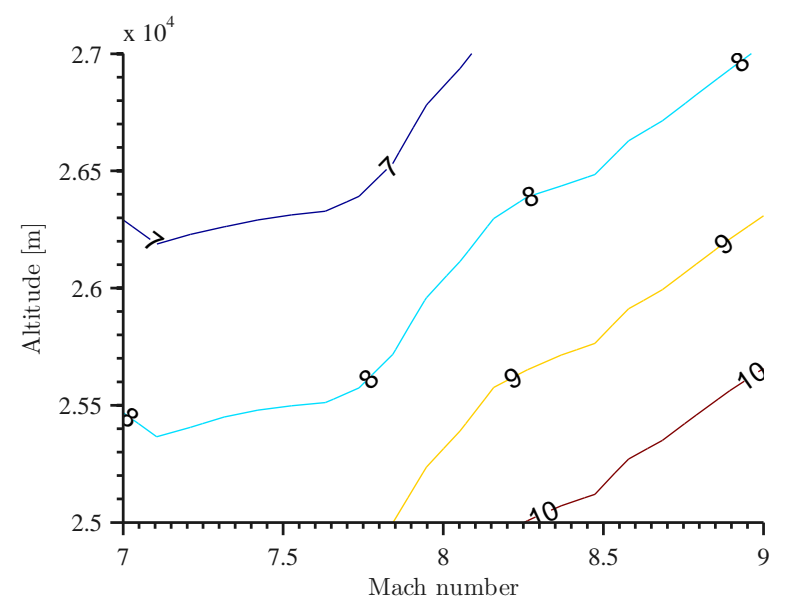

a) Operating map, $\phi=0.1$

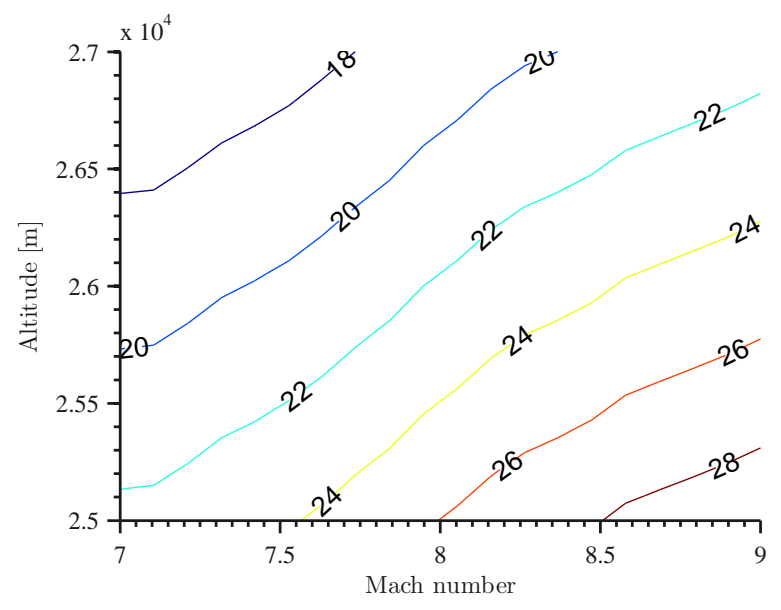

c) Operating map, $\phi=0.3$

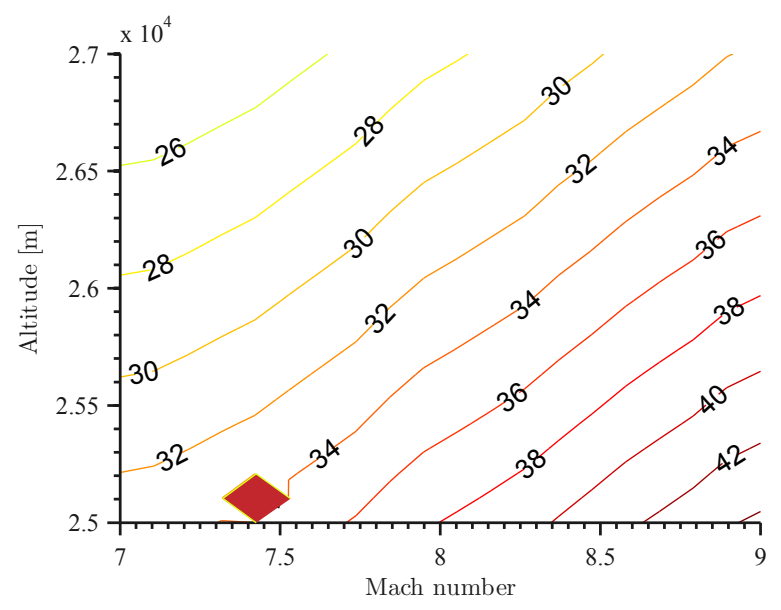

e) Operating map, $\phi=0.5$

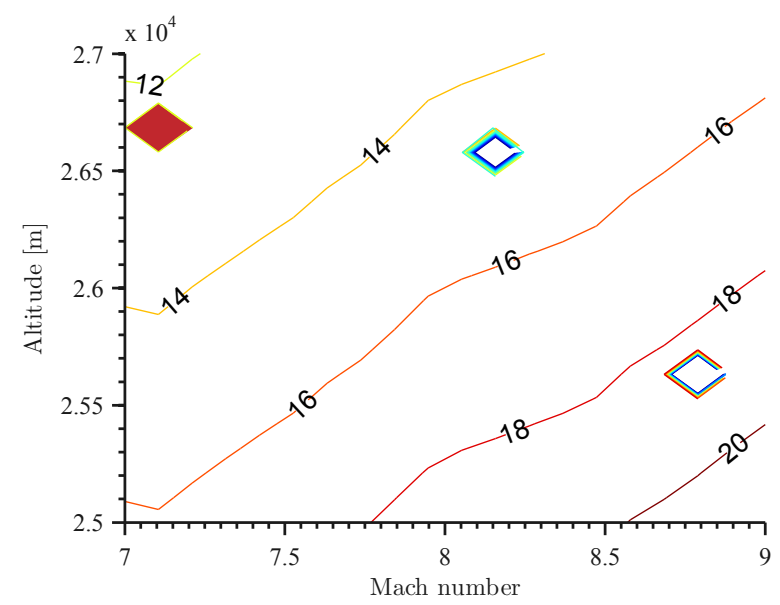

b) Operating map, $\phi=0.2$

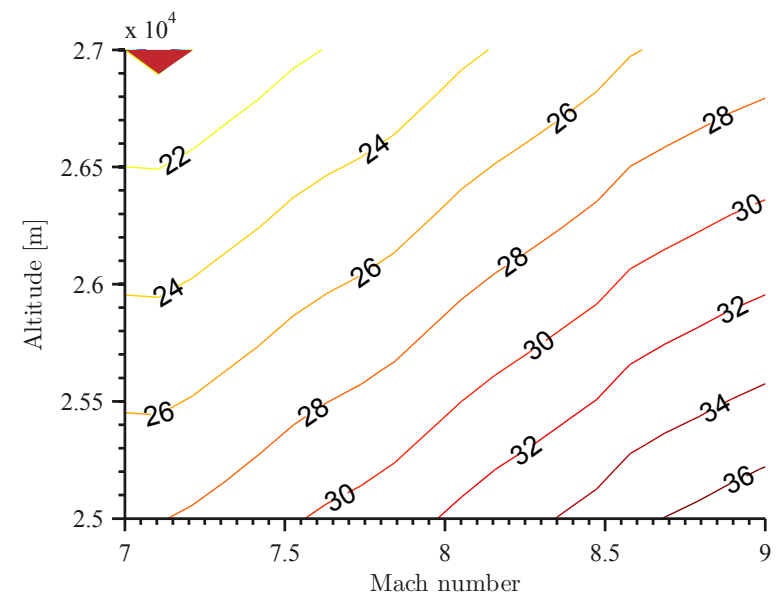

d) Operating map, $\phi=0.4$

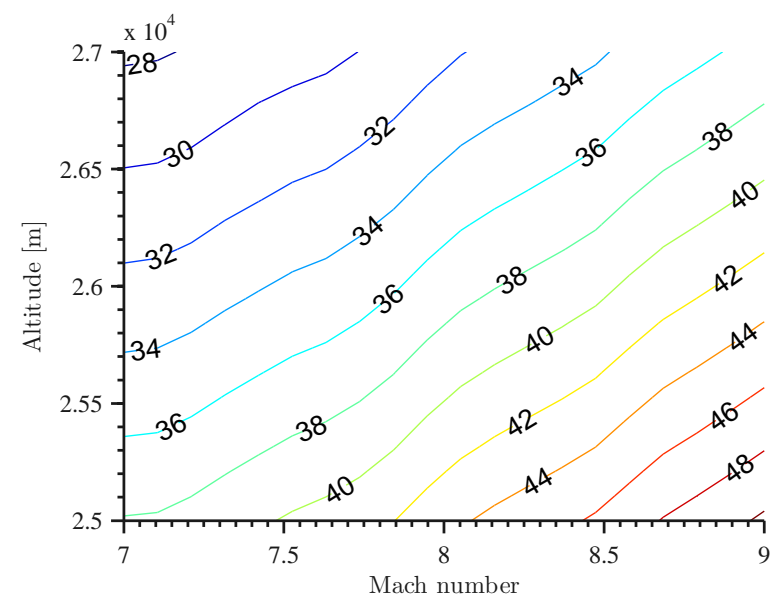

f) Operating map, $\phi=0.6$

Figure 10. Operating maps for $\phi=0.1$ to 0.6. Thrust contours are given in $\frac{\mathrm{kN}}{\mathrm{m}}$. Red marks identify points at which choking may occur. 